\title{
Discovery of New Be Stars in the Galactic Open Cluster NGC7128
}

\author{
Z. Balog ${ }^{1,2,3}$
}

Harvard-Smithsonian Center for Astrophysics 60 Garden Street, Cambridge MA, 02138 USA

\section{J. Vinkó ${ }^{3}$}

Magyary Postdoctoral Fellow, Dept of Optics, JATE University, H-6720 Dóm tér 9. Szeged, Hungary

\section{G. Fürész ${ }^{1}$}

Dept of Exp. Physics, JATE University, H-6720 Dóm tér 9. Szeged, Hungary

\section{E. J. Alfaro, A. J. Delgado and A. Moitinho}

Instituto de Astrofísica de Andalucia, Camino Bajo de Huetor, 24 E-18008 Granada, Spain

\begin{abstract}
We present medium resolution spectroscopy $(\lambda / \Delta \lambda \approx 7,000)$ covering $6500-6750 \AA$ of three emission stars in the highly reddened young open cluster NGC7128. Two of them were previously unknown. The known Be star (V1814 Cyg) is an irregular variable; one of the newly discovered stars is an eclipsing binary (V1481 Cyg) (Jerzykiewicz et al., 1996.). Physical parameters of the cluster have been determined from new $U B V$ and $u v b y \mathrm{H} \beta$ photometric measurements.
\end{abstract}

\section{Introduction}

The aim of this paper is to report the discovery of new Be stars in the open cluster NGC7128. Only one Be star was previously known in this open cluster (V1814 Cyg) which is a known variable (Jerzykiewicz et al., 1996.) One of the new Be stars shows definite $\mathrm{H} \alpha$ emission; the other is a known eclipsing binary (V1481 Cyg) has a more complicated line profile. The discovery of Be stars in a cluster with well determined physical parameters allows estimates for the age

\footnotetext{
${ }^{1}$ Visiting Astronomer, German-Spanish Astronomical Centre, Calar Alto, operated by the MaxPlanck-Instiute for Astronomy, Heidelberg, jointly with the Spanish National Commission for Astronomy.

${ }^{2}$ Visiting Astronomer, David Dunlap Observatory, Richmond Hill, Ontario, Canada

${ }^{3}$ on leave from Dept. of Optics JATE University, H-6720 Dóm tér 9. Szeged, Hungary
} 
and intrinsic colors of the Be stars for comparison with theoretical models for the evolutionary status of Be stars.

\section{Observations}

We have obtained medium resolution $(\lambda / \Delta \lambda \approx 7,000)$ spectroscopic observations covering $6500-6750 \AA$ of the 12 brightest stars in the field of NGC7128. This spectral region contains the $\mathrm{H} \alpha$ and the He I $\lambda 6678$ line. The observing program was carried out on 10-11 July 1998 at David Dunlap Observatory. We used the Cassegrain spectrograph attached to the $1.88 \mathrm{~m}$ telescope. The exposure times were about 30-40 minutes. The spectra were reduced with standard IRAF routines. For wavelength calibration two FeAr spectral lamp exposures were recorded for every stellar spectrum. $U B V$ and $u v b y \mathrm{H} \beta \mathrm{CCD}$ observations were also performed at Calar Alto (17th July 1996, 9th July 1997), Roque de los Muchachos (7-8th August 1996) and San Pedro Mártir to measure the physical parameters of the cluster. These are listed in Table 1 (Balog \& al. in preparation).

Table 1. Physical Parameters of NGC7128

$\begin{array}{lc}\log \text { (Age) } & 7.4 \\ \text { Distance modulus [mag] } & 12.65 \\ E(B-V)[\mathrm{mag}] & 1.07 \\ E(b-y)[\mathrm{mag}] & 0.73\end{array}$

\section{Results}

We derived the physical parameters of the Be stars \#4 (V1814 Cyg) and \#1081 (Fig. 1a-b) from the cluster parameters and spectroscopic observations. The numbering system is from Mermilliod's Open Star Cluster Database (WEBDA, http://obswww.unige.ch/webda).

We measured the cluster parameters using isochrone fitting (Balog \& al. in preparation) and the latest isochrones of Claret (1999 personal communication) assuming solar metallicity. We estimated spectral types of the emission stars from their dereddened color indices and the presence/absence of He I $\lambda 6678$ line in their spectra. The spectrum of \#4 contains this line, and therefore probably has an early B spectral type. The spectrum of \#1081 does not show the He I line, which indicates a late-B spectral type. We assigned masses and effective temperatures of the two stars from their positions on the fitted isochrone. The photometric indices suggest that these stars are subgiants. Our results support the spectral classification of Svolopoulos (1961) for the star \#4 (B3 IV). We estimated the extra reddening due to circumstellar matter around Be-stars using the calibrations of Fabregat \& Torrejon (1998). This calibration is valid only for stars earlier than B5; we thus estimated the extra reddening only for \#4. Table 2. lists the physical parameters of the two obvious Be stars. 

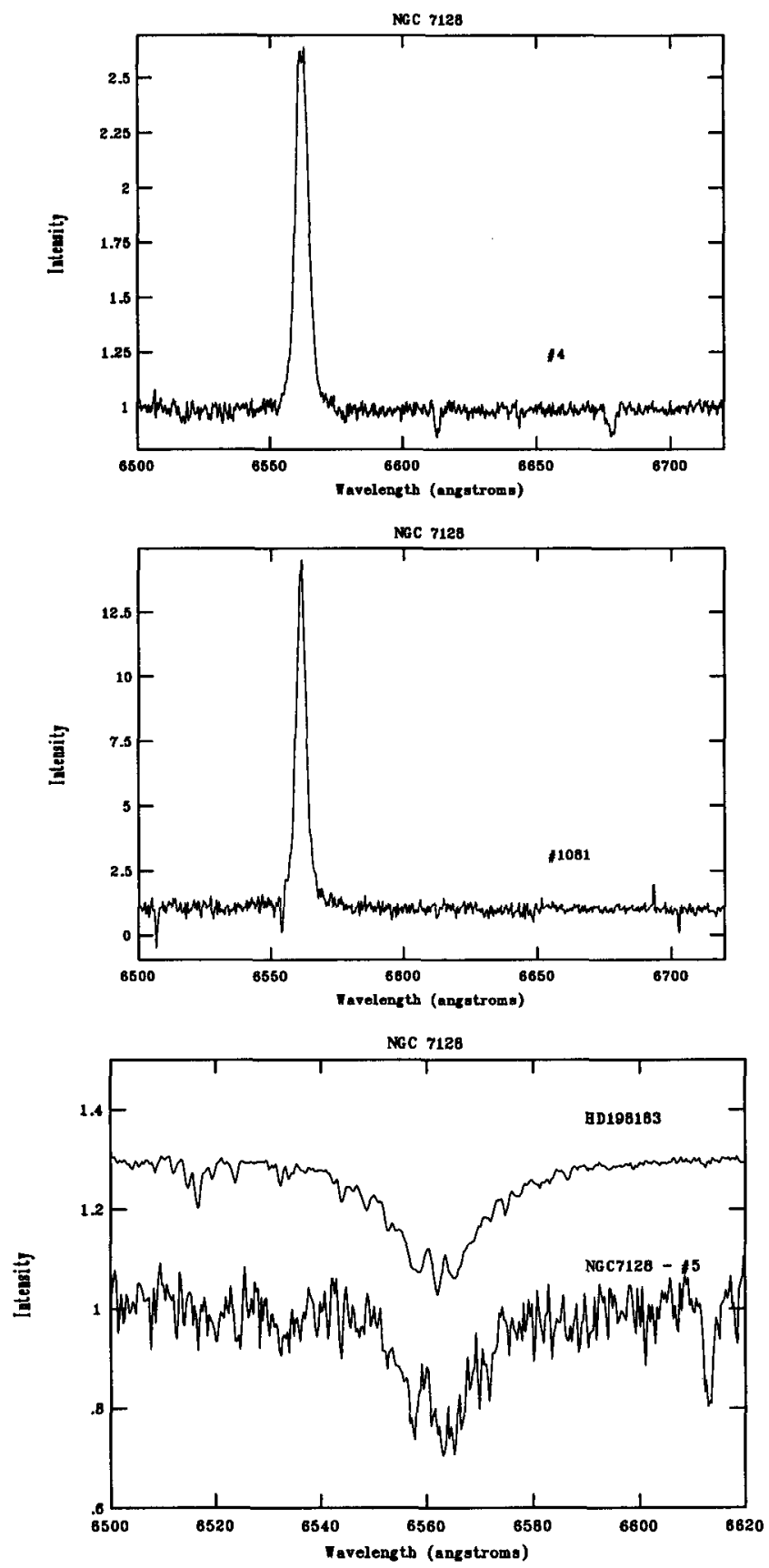

Figure $1 . \quad$ a-c) The spectrum of star \#4 (V1814 Cyg), \#1081, \#5 (V1481 Cyg) of NGC7128. In panel c (\#5) we also plot the spectrum of the known emission star HD198183 for comparison. 
Table 2. Physical parameters of the two obvious Be stars

\begin{tabular}{lcc} 
Star & $\# 4$ (V1814 Cyg) & $\# 1081$ \\
\hline EW of H $\alpha[\AA]$ & -11 & -59 \\
Spectral Type & early B & late B \\
$T_{\text {eff }}[\mathrm{K}]$ & $18300 \pm 4000$ & $20100 \pm 4000$ \\
\left.${\text { Mass }\left[\mathrm{M}_{\odot}\right]}_{M_{\mathrm{v}}[\mathrm{mag}]}\right]$ & -4.5 & 8.7 \\
$(b-y)_{0}[\mathrm{mag}]$ & -0.07 & -2.7 \\
$(B-V)_{0}[\mathrm{mag}]$ & -0.23 & -0.09 \\
$E(b-y)[\mathrm{mag}]$ & 0.029 & -0.22 \\
$E c s(c 1)[\mathrm{mag}]$ & -0.052 & - \\
& & -
\end{tabular}

The Be star nature of V1481 Cyg (star \#5 of NGC7128) is suspected from the similarity of its $\mathrm{H} \alpha$ with that of HD198183, a known Be-star (Fig. 1c). However, V1481 Cyg is also a binary system, which makes its line profiles complicated. Further observations are therefore needed to confirm the emission component in its hydrogen lines.

Acknowledgments. This work was supported by the SOROS Fundation, the Foundation for Hungarian Education and Science and the Hungarian OTKA Grants \#F022249 and \#T022259.

\section{References}

Fabregat, J., Torrejon, J.M. 1998, A\&A 332, 643

Jerzykiewicz, M., Pigulski, A., Kopacki, G., Mialkowska, A., Niczyporuk, S. 1996, Acta Astron 46, 253

Svolopoulos, S.N. 1961, ApJ 134, 612 